\title{
Determinismo Tecnológico: elementos para debates em perspectiva educacional $^{1}$
}

\author{
Technological Determinism: elements for debates on educational perspective
}

\author{
Raquel Folmer Corrêa ${ }^{2}$ \\ Bethania Medeiros Geremias ${ }^{3}$
}

\begin{abstract}
RESUMO
Neste artigo, apresentamos alguns elementos para debater a temática do determinismo tecnológico sob um ponto de vista voltado a questões educacionais. Com um suporte teórico baseado sobretudo nos estudos latino-americanos que relacionam ciência, tecnologia e sociedade (CTS), o objetivo é pensar o determinismo tecnológico como algo central para problematizar questões tecnocientíficas em sala de aula. $O$ artigo tem caráter teórico e nele realizamos uma revisão bibliográfica na qual abordamos significados e origens do termo determinismo tecnológico, apresentamos autores relacionados a essa perspectiva, propomos a incorporação de estudos sobre processos educacionais aos debates sobre o tema e apresentamos uma reflexão crítica final. Com isso, resultam possibilidades de superar visões limitantes das inter-relações entre conhecimentos, técnicas e coletivos. A percepção crítica de tais inter-relações é considerada aqui como algo fundamental para pensarmos e propormos processos educativos que contemplem uma formação humana crítica, integral e permanente.
\end{abstract}

Palavras-chave: Determinismo tecnológico. Tecnologia. Estudos CTS.

\begin{abstract}
This article presents some elements to discuss the issue of technological determinism from a point of view facing the educational issues. With a theoretical framework based mainly in Latin American studies that relate science, technology and society (STS), the goal is to think of technological determinism as something central to problematize technoscientific issues in classroom. The paper is theoretical and shows a literature review in which we discuss meanings and origins of the term technological determinism, we present authors related to this perspective, we propose the incorporation of studies on educational processes to debates on the subject and present a final critical reflection. From this, arise possibilities to overcome limited viewpoints of the inter-relationships between knowledge, skills and collective. The critical perception of such interrelationships is seen here as something essential to think and propose educational processes that include a human critical, integral and permanent.
\end{abstract}

Keywords: Technological determinism. Technology. STS studies.

\footnotetext{
${ }^{1}$ Trabalho apresentado no dia 17 de outubro de 2013, no Grupo de Trabalho 16 - Educação Científica e Tecnológica e Estudos CTS: Novos Desafios e Possibilidades.

${ }^{2}$ Doutoranda no Programa de Pós-Graduação em Educação Científica e Tecnológica pela Universidade Federal de Santa Catarina (PPGECT/UFSC) Bolsista CAPES; Mestre em Sociologia pelo Programa de Pós-Graduação em Sociologia da Universidade Federal do Rio Grande do Sul (PPGS/UFRGS); Bacharel e Licenciada em Ciências Sociais pela UFRGS. E-mail: raqfolmer@hotmail.com.

${ }^{3}$ Doutoranda no Programa de Pós-Graduação em Educação Científica e Tecnológica; Mestre em Educação Científica e Tecnológica (PPGECT/UFSC); Bolsista do Programa do Observatório da Educação (OBEDUC/CAPES); Mestre em Educação (Université de Nantes/França); Graduada em Pedagogia. E-mail: bmgeremias@gmail.com.
} 


\section{INTRODUÇÃO}

Neste artigo, temos o foco em questões relacionadas a visões deterministas sobre a tecnologia e em possibilidades de problematização dessas visões em âmbito educacional. Dentro dos estudos latino-americanos que relacionam ciência, tecnologia e sociedade (CTS), em especial dos estudos em Educação CTS, destacamos o determinismo tecnológico como um dos principais entraves para se estabelecer propostas de processos educacionais críticos em relação a questões sócio-tecno-científicas.

Tais processos educacionais críticos podem ser abordados desde a perspectiva discursiva de Educação CTS (CASSIANI e LINSINGEN, 2010), que busca contribuições da Análise de Discurso de linha francesa, dos Estudos CTS latino-americanos e das abordagens de educação crítica, como as de Paulo Freire (1921-1997), para construir processos formativos que possibilitem "maior inserção social das pessoas no sentido de se tornarem aptas a participarem dos processos de tomadas de decisões conscientes e negociadas em assuntos que envolvam ciência e tecnologia" (LINSINGEN, 2007, p. 13).

Defendemos que análises críticas sobre processos educacionais (formais, não formais ou informais) não são possíveis através da continuidade de mentalidades deterministas. Compreende-se que é insuficiente considerar que empreendimentos tecnológicos determinem, necessariamente, o desenvolvimento econômico e social, na medida em que não é possível garantir que o incremento tecnológico se converta em progressos para a sociedade ou em melhoria social, nem que as tecnologias guardem uma dimensão de determinação sobre as sociedades.

A seguir, serão abordadas as origens e os significados do termo determinismo tecnológico. Depois, serão discutidos autores relacionados a esse tema, assim como a processos educacionais e determinismo tecnológico e, por fim, serão encaminhadas as reflexões finais. 


\section{SIGNIFICADOS E ORIGENS DO TERMO "DETERMINISMO TECNOLÓGICO"}

O determinismo tecnológico se refere a uma ideia bastante popular sobre as relações entre tecnologia e sociedade e que, segundo Chandler (1995), caracteriza uma linha de pensamento influente na atualidade. A expressão "determinismo" teria sido utilizada inicialmente pelo sociólogo e economista americano Thorstein Veblen (1857-1929), em textos nos quais esse autor trata das relações entre o automatismo técnico e os mercados capitalistas (ELLUL, 1964).

Contudo, o conteúdo presente na perspectiva do determinismo tecnológico tem seu início relacionado ao auge da ideia de progresso (CHÁVARRO, 2004). Até o final do século XIX, a crença nos avanços tecnológicos e sua determinação no bem estar humano já havia se tornado um dogma, pois que "una de las asociaciones más frecuentes con el término tecnología es la idea de progreso, hasta el grado de ser uma representación social en las mentalidades colectivas" (idem, p.137).

Conforme a historiadora Montserrat Huguet (2003), o sentido de progresso ad infinitum se incorporou à história ocidental inteiramente no século XIX, sendo o século XX herdeiro destacado de uma proposta ideológica e social que legitima 0 crescimento tecnológico como sinônimo de desenvolvimento e incorpora, ao mesmo tempo, dimensões de gigantismo e aceleração jamais imaginadas.

Através da concepção de determinismo tecnológico, tenta-se explicar fenômenos sociais e históricos de acordo com um fator principal que, no caso, seria a tecnologia. Feenberg (1991) esclarece que o determinismo se baseia na suposição de que as tecnologias teriam uma lógica funcional autônoma que poderia ser explicada sem se fazer referência à sociedade.

$\mathrm{Na}$ perspectiva determinista considera-se que 0 desenvolvimento tecnológico condiciona sobremaneira a mudança e as estruturas sociais. Como a fonte primordial das mudanças sociais se caracterizaria pelo desenvolvimento de tecnologias, as intervenções sociais, políticas, institucionais e culturais colocadas ao processo de desenvolvimento de tecnologia teriam pouco ou nenhum efeito. Isso ocorreria porque as tecnologias afetariam inexoravelmente todos os âmbitos sociais (CHANDLER, 1995).

Segundo a concepção determinista da tecnologia, considera-se a relação entre tecnologia e sociedade como unidirecional, sustenta-se que o desenvolvimento 
social, em seus aspectos econômicos, políticos e culturais, seja uma consequência direta e linear do desenvolvimento tecnológico. Sob tal ponto de vista, argumenta-se que a tecnologia segue um curso particular de acordo com as suas próprias leis e se desenvolve num âmbito externo ao meio social, como um fator exógeno, com uma dinâmica própria, como se fosse um fenômeno natural que responde aos seus próprios princípios e ao qual ao homem só restaria o esforço por adaptar-se (idem).

Diéguez (2005) aponta que a perspectiva determinista se relaciona às ideias de autonomia, condicionamento e unidirecionalidade. A tecnologia se desenvolveria de modo autônomo e independente, ela seria o fator que condicionaria o desenvolvimento da sociedade. A relação entre tecnologia e sociedade ocorreria de modo unidirecional, daquela agindo sobre esta. Mesmo que tais características sejam analisadas de modo independente por alguns autores que examinam a questão, elas parecem encerrar o conteúdo central de determinismo, tal como ele é aqui analisado, a saber, que o determinismo tecnológico pode ser examinado como a ausência de conhecimento e de controle dos processos que envolvem as questões relacionadas à tecnologia (desenvolvimento, produção, educação, divulgação e apropriação) por parte da sociedade.

Tendo em vista certas características e peculiaridades da questão, destacamos a interpretação de determinismo tecnológico apresentada por Smith e Marx (1994). Segundo esses autores podemos verificar que

(...) a ideia de determinismo tecnológico assume diversas formas, que podem ser descritas como algo que ocupa espaços ao longo de um espectro entre extremos "hard" e "soft". No extremo "hard" do espectro, a atividade (o poder de efetuar mudança) é imputada à tecnologia a si mesma ou a alguns dos seus atributos intrínsecos (...). No outro extremo do espectro, o determinismo "soft" começa por recordar-nos que a história da tecnologia é uma história de ações humanas. Para entender a origem de um tipo particular de poder tecnológico, devemos primeiramente aprender sobre os atores. Quem eram eles? Quais foram as circunstâncias? (...) Por que a inovação foi feita por essas pessoas e não por outras? Por que foi possível nesse momento e nesse lugar, em vez de em outro tempo ou lugar? Quem se beneficiou, e quem sofreu? (...) Em vez de tratar a "tecnologia" per se como o locus da ação histórica, os deterministas "soft" localizam-na em uma matriz mais diversa e complexa do ponto de vista social, econômico, político e cultural (ibidem, p. 12-13, tradução livre).

O determinismo mais leve (soft) consideraria como agente causal uma matriz social, econômica, política e cultural variada e complexa, e não apenas a tecnologia. O determinismo mais forte (hard) atribuiria à tecnologia o poder de provocar mudança social segundo um caráter inevitável e necessário. 
Para além dessas características evidenciadas nas perspectivas de determinismo tecnológico, relacionamos brevemente a seguir autores que se considera (não sem provocar desacordos) terem relação com essa visão.

\section{AUTORES RELACIONADOS AO DETERMINISMO TECNOLÓGICO}

O determinismo tecnológico tem sido atribuído, com maior ou menor justiça, a autores variados. Sem pretender simplificar de maneira inadequada as abordagens complexas propostas pelos seguintes autores, examinamos brevemente aspectos pontuais de interpretações deterministas em Karl Marx (1818-1883), Jacques Ellul (1912-1994) e Herbert Marcuse (1898-1979) 4 .

Para Chandler (1995), a posição de Jacques Ellul parece ser radical, pois, para Ellul, o domínio da tecnologia iria além da técnica e constituiria a dominação da vida por critérios da lógica e da eficiência. Ellul (1964) enfatiza que a tecnologia carrega consigo seus próprios efeitos, independentemente de como é usada. As tecnologias carregariam um número de consequências positivas e negativas, não importando como e para que sejam utilizadas. Não seria apenas uma questão de intenções.

A técnica condiciona e provoca as mudanças sociais, políticas e
econômicas. É o motor de todo o resto, apesar das aparências e apesar do
orgulho do homem, que pretende que suas teorias filosóficas ainda tenham
uma força determinante e que seus regimes políticos sejam decisivos na
evolução técnica. Não são mais as necessidades externas que determinam
a técnica, são suas necessidades internas. Tornou-se uma realidade em si,
que se basta a si mesma, com suas leis particulares e suas determinações
próprias (idem, p. 133-134, tradução livre).

Para Ellul, a tecnologia seria autônoma e seguiria suas próprias leis de desenvolvimento, com total independência dos desejos humanos e de qualquer outro fator externo.

Já o posicionamento de Marx em relação à tecnologia causa intensos debates. No segundo capítulo de seu texto "Miséria da Filosofia", de 1847, Marx faz

\footnotetext{
${ }^{4} \mathrm{~A}$ escolha desses autores se deve tanto à importância de suas abordagens acerca das questões tecnológicas quanto ao caráter de suas argumentações em relação a entendimentos de relações entre sociedade e tecnologia.
} 
algumas observações e levanta contradições quanto à explicação do método econômico-metafísico do anarquista francês Proudhon (1809-1865).

O Sr. Proudhon, o economista, compreendeu muito bem que os homens fabricam o pano, a fazenda, os tecidos de seda em determinadas relações de produção. Mas o que ele não compreendeu, é que essas relações sociais determinadas são também produzidas pelos homens, do mesmo modo que a fazenda, o linho, etc. As relações sociais estão intimamente ligadas às forças produtivas. Adquirindo novas forças produtivas, os homens mudam o seu modo de produção e, mudando o modo de produção, a maneira de ganhar a vida, mudam todas as relações sociais. O moinho manual dar-vos-á a sociedade com o suserano; o moinho a vapor, a sociedade com o capitalismo industrial. Os homens que estabelecem as relações sociais de acordo com a sua produtividade material, produzem também os princípios, as ideias, as categorias, conforme as suas relações sociais. Assim, estas ideias, estas categorias, são tão pouco eternas como as relações que exprimem. São produtos históricos e transitórios (MARX, 1990 , p. 121-122).

A declaração de Marx de que "o moinho manual dar-vos-á a sociedade com o suserano; o moinho a vapor, a sociedade com o capitalismo industrial" (idem, ibidem) veio a ser interpretada como uma aceitação do determinismo tecnológico (CHÁVARRO, 2004; DAGNINO, 2008).

No entanto, como destaca Dagnino (2008), nos textos de Marx há passagens nas quais a tecnologia pode ser compreendida como um elemento neutro, simplesmente instrumental. Nessa situação, o importante seria a apropriação da tecnologia pela classe operária. Em outras passagens, podem-se verificar afirmações nas quais a tecnologia é compreendida como trazendo em si, de modo inerente, um elemento de subordinação e maior exploração do trabalhador.

Para além das controvérsias acerca da posição de Marx em relação à tecnologia, consideramos a perspectiva de Dagnino (2008), segundo o qual nenhum outro cientista social de importância conferiu tanta relevância à mudança tecnológica como Marx. Para esse autor, a obra de Marx se configura com um marco na forma de se estudar os desenvolvimentos tecnológicos, pois abandona a forma tradicional, centralizada na figura do inventor singular e seu "gênio". Marx propõe uma abordagem social da tecnologia e, ao mesmo tempo, incorpora o desenvolvimento tecnológico como constitutivo das explicações históricas (idem).

Marcuse também desperta interpretações segundo as quais ele teria aceitado o determinismo tecnológico ao destacar a vida unidimensional do homem contemporâneo, fruto de uma racionalidade tecnológica (CHÁVARRO, 2004). No texto "A ideologia da sociedade industrial", de 1964, Marcuse demonstra novas 
formas de dominação existentes nas sociedades industriais avançadas. Para o autor, entre outras coisas, os avanços tecnológicos dessas sociedades seriam os responsáveis pelo sistema totalitário de dominação.

Tal sistema de dominação prescreveria uma nova ideologia de imposição de certa racionalidade institucional ou tecnológica em relação à racionalidade individual, o que submeteria o homem a uma completa alienação. A racionalidade tecnológica causaria certo conformismo, no qual qualquer tipo de manifestação individual revolucionária dentro de uma sociedade totalmente planejada seria inapropriado.

(...) no período contemporâneo, os controles tecnológicos parece serem a
própria personificação da Razão para o bem de todos os grupos e
interesses sociais - a tal ponto que toda contradição parece irracional e toda
ação contrária parece impossível. Não é, portanto, de admirar que, nos
setores mais desenvolvidos dessa civilização, os controles sociais tenham
sido introjetados a ponto de até o protesto individual ser afetado em suas
raízes (MARCUSE, 1969, p. 30).

De acordo com Trigueiro (2008), para Marcuse, não haveria saída para uma ciência e uma tecnologia emancipadoras segundo as estruturas do modo de produção capitalista. O estabelecimento de uma nova ciência e uma nova tecnologia prescreveria uma nova estrutura social e um modo diferenciado de encarar a natureza e as relações entre os indivíduos, livres da dominação e do controle de uns sobre outros. A crítica à tecnologia deveria passar pela crítica à própria sociedade e ao modelo de ciência e tecnologia correspondentes ao capitalismo.

Essas breves referências a obras e reflexões dos autores citados acima permite que se compreenda como o determinismo tecnológico é atribuído controversamente a pensadores diferentes em seus contextos específicos. Com isso, nossa intenção é propiciar subsídios para debates críticos sobre o determinismo tecnológico tendo em vista, também, perspectivas educacionais.

\section{DETERMINISMO TECNOLÓGICO E PROCESSOS EDUCACIONAIS}

Existem diversos modos de olhar a questão do determinismo tecnológico. Destacamos que há uma dimensão cultural significativa que pode ser considerada nesse debate. De acordo com Chávarro (2004), os professores Wiebe Bijker (1951-) e Trevor Pinch (1952-) têm defendido uma orientação contrária à perspectiva 
determinista, qual seja, a da construção social. Esses autores trabalham com a ideia de que as forças sociais e culturais também determinam a mudança técnica. Em múltiplos estudos, eles têm mostrado como a tecnologia é uma construção social e, para isso, Bijker desenvolve o conceito de marco tecnológico. Tal conceito se centra nos significados que os grupos sociais atribuem a um artefato e na gramática que se desenvolve ao redor desses para explicar como o ambiente social estrutura o desenho de um artefato.

De nossa parte, ao debatermos sobre determinismo tecnológico, além do foco nas dimensões culturais, consideramos possibilidades de superar esse tipo de visão, que limita os entendimentos sobre as inter-relações entre diversos conhecimentos, técnicas e coletivos. Como dissemos anteriormente, compreendemos que uma percepção crítica dessas inter-relações é algo fundamental para a elaboração e execução de processos educativos que contemplem uma formação humana crítica, integral e permanente.

Com as discussões propostas neste artigo, reforçamos a intenção, semelhante a presente em Cassiani e Linsingen (2010), de que os estudos CTS abram-se cada vez mais aos temas educacionais. Entendemos que a superação das premissas do determinismo tecnológico passa, também, pelos planos educacionais (educação politécnica, por exemplo), de modo que seja possível problematizar questões tecnocientíficas de maneira crítica, participativa e colaborativa na sala de aula e em diferentes espaços educacionais.

Nas perspectivas educacionais dos estudos CTS latino-americanos, em autores como Linsingen (2007), defende-se que uma abordagem educacional deve ser contextualizada, em sintonia com os aspectos sociais e comprometida em termos curriculares. Em conformidade com isso, propomos a incorporação de debates sobre determinismo tecnológico nos estudos sobre processos educacionais, pois a educação CTS pode considerar, também, "questões que envolvem os variados aspectos das relações sociais e econômicas regionais, abarcando o campo das políticas públicas de C\&T com suas percepções de relevância" (idem, p. 02). 


\section{REFLEXÕES FINAIS}

Neste artigo, destacamos aspectos do determinismo tecnológico para que se possam verificar implicações desse tipo de visão no que se refere a perspectivas educacionais. A intenção foi chamar a atenção para uma visão crítica da tecnologia na qual essa seja percebida como um fenômeno imerso em condicionamentos socioculturais, econômicos e políticos.

Criticar o determinismo tecnológico, no entanto, não significa, absolutamente, promover uma posição voluntarista. A intenção foi verificar como esse tipo de pensamento, que é atribuído de modo controverso a diferentes pensadores, representa uma visão redutora dos relacionamentos entre o desenvolvimento social e tecnológico. Pensar que a tecnologia, por si mesma, é capaz de modificar o ser humano, seus hábitos e instituições, pode encobrir a possibilidade de adaptação ou resistência às contingências históricas e às formas com que diferentes coletividades se relacionam diariamente com as tecnologias.

Frente a isso, retomamos nossa consideração de que o determinismo tecnológico pode ser examinado como a ausência de conhecimento/controle de processos que envolvem questões tecnocientíficas por parte da sociedade e propomos uma ampliação das compreensões e debates sobre o tema, sobretudos nos diversos espaços escolares. Assim, esperamos poder contribuir para a formulação de agendas de pesquisas nas quais se considerem que as diversas coletividades possam ter acesso, conhecimento e possibilidade de escolha crítica nos processos que envolvem questões relacionadas ao desenvolvimento, produção, divulgação educação e apropriação de tecnologias.

\section{REFERÊNCIAS}

BIMBER, B. Three Faces of Technological Determinism. In: SMITH, M. R.; MARX, L. Does Technology Drive History? The Dilemma of Technological Determinism. Massachusetts: MIT, 1994.

CASSIANI, S. e LINSINGEN, I. von Educação CTS em perspectiva discursiva: contribuições dos Estudos Sociais da Ciência e da Tecnologia. Redes, Buenos Aires. V. 16, no 31 , dezembro de 2010, p. 163-182. 
CHANDLER, D. Technological or Media Determinism. (1995) Disponível em: $<$ http://www.aber.ac.uk/media/Documents/tecdet/tecdet.html> Acessado em abril de 2009.

CHÁVARRO, L. A. El debate sobre el determinismo tecnológico: de impacto a influencia mutua. S\&T - Sistemas e telemática da Universidade ICESI, 2004, p.121 143. Disponível em: <http://openpdf.com > . Acesso em: março de 2009.

DAGNINO, R. Neutralidade da ciência e determinismo tecnológico. Campinas: Unicamp, 2008.

DIÉGUEZ, A. El determinismo tecnológico: indicaciones para su interpretación. Argumentos de Razón Técnica, V.8, 2005, p. 67-87. Disponível em:

<http://openpdf.com/>. Acesso em: março de 2009.

ELLUL, J. The Technological Society. New York: Vintage Books, 1964.

FEENBERG. A. Racionalização subversiva: tecnologia, poder e democracia. (1991) Disponível em: <http://www.sfu.ca/ andrewf/languages.html>. Acesso em: agosto de 2009.

HUGUET, M. El determinismo tecnológico ¿Un nuevo discurso legitimador? Claves de Razón Práctica. n¹34, julio/agosto 2003, p. 3145.

LINSINGEN, I. von. Perspectiva educacional CTS: aspectos de um campo em consolidação na América Latina. Ciência \& Ensino, vol. 1, número especial, novembro de 2007, p. 01-19.

MARCUSE, H. A ideologia da sociedade industrial. Rio de Janeiro: Zahar, 1969.

MARX, K. Miséria da filosofia. São Paulo: Mandacaru, 1990.

SMITH, M. R.; MARX, L. Does Technology Drive History? The Dilemma of Technological Determinism. Massachusetts: MIT, 1994.

TRIGUEIRO, M. O conteúdo social da tecnologia. Brasília: Embrapa Informação Tecnológica, 2008. 